\title{
Should patients with stage II colon cancer be treated with adjuvant chemotherapy?
}

\author{
Andrzej Deptała
}

Patients with stage II colon cancer, as defined by pTNM/AJCC 2017, when analysed with regards to the risk of recurrence and to DFS and OS prolongation with adjuvant chemotherapy, make up a heterogenous patient population. That is why, a qualified oncologist, who makes a decision to apply adjuvant chemotherapy, taking into consideration the patient's well-being, should formulate a post-operative strategy, the main objective of which will be to minimise the risk of cancer recurrence and to prolong survival.

The author of this paper believes that adjuvant chemotherapy should be provided for every patient in the very high risk group (IIC) and high risk group (IIA/IIB MSI-L/MSS and inadequate lymphadenectomy and BRAF mutation), and also in the group of intermediate risk of cancer recurrence (IIA/IIB MSI-L/MSS with an adequate scope of lymphadenectomy and BRAF mutation as well as IIA MSI-L/MSS with inadequate scope of lymphadenectomy and lack of BRAF mutation), after obtaining the patient's informed consent. In a situation when an oncologist is unable to obtain the result of microsatellite instability or the BRAF gene mutation, it would be advisable to refer the patient to a centre which has access to such tests; this would allow the adjuvant chemotherapy to have optimum application, which would by all means translate into the improvement of the treatment results in patients with colon cancer.

NOWOTWORY J Oncol 2017; 67, 6: 380-384

Key words: colon cancer, stage II in pTNM/AJCC, adjuvant chemotherapy, microsatellite instability

\section{Introduction}

Colorectal cancer (CRCA) belongs to the most frequently occurring malignant cancers, both in Poland and worldwide. The tumour is most frequently located in the colon (C18 - colon cancer), understood by oncologists as a sum of the consecutive anatomical parts of the large intestine (caecum - C18.0, ascending colon - C18.2, hepatic flexure - C18.3, transverse colon - C18.4, splenic flexure - C18.5, a descending colon - C18.6, sigmoid colon - C18.7, sigmoido-rectal flexure - C19). Rectal cancer (C20), although also constituting a colorectal cancer, is treated separately by oncologists from the point of view of the differences in therapeutic management. Appendix cancer (C18.1) and anal cancer (C21) are the tumours with a completely different biology, and thus require a different treatment strategy; therefore, in oncological nomenclature, they are not included in the category of colorectal cancer [1].
A basic treatment method of colon cancer without distant metastases seen in diagnostic imaging (M0) is a surgical resection. However, about a half of the patients after radical resection of colon cancer will develop metastases within the next 5 years. This is due to the fact that in many cases the advancement stage of colon cancer, determined on the basis of the histopathological assessment of the post-operative specimen, is higher than the stage established on the basis of the diagnostic imaging. In $25 \%$ of cases with a clinically early cancer, a pathomorphologist is able to find some infiltration of pre-serous colon tissues (pT3) or visceral peritoneum ( $\mathrm{pT} 4 \mathrm{a}$ ) or neighbouring tissues and organs (pT4b), and, moreover, in 30-40\% of cases a pathomorphologist is able to find metastases in the regional lymph nodes (pN1a-b, pN2a-b stages), which were not found before the surgery [2]. That is why, in such cases, the main strategy in post-operative therapy is the treatment oriented towards 
minimising the risk of cancer recurrence. This role, in the treatment of colon cancer, should be played by adjuvant chemotherapy.

\section{The role of adjuvant chemotherapy}

The objective of adjuvant chemotherapy (which completes the surgical resection) is to annihilate all cancer cells and micro-metastases in order to prevent the development of clinically overt distant metastases and to prolong disease-free survival (DFS), relapse-free survival (RFS), disease-specific survival (DSS) and overall survival (OS) [1].

Adjuvant chemotherapy may be taken into consideration when a pathologist does not find any distant metastases in the post-operative specimen (pM0) and when the tumour resection was $\mathrm{RO}$, i.e. was both microscopically and macroscopically radical. Radical resection means that the scope of the lymphadenectomy was optimal, i.e. at least 12 regional lymph nodes draining lymph from a specific section of the colon were removed by a surgeon and then assessed by a pathomorphologist under a microscope [1, 2].

\section{Adjuvant chemotherapy in stage II colon cancer}

The fact that colon cancer patients benefit from the 5-FU-based adjuvant chemotherapy was confirmed already in the 1980s and 1990s. A collective analysis published in 2004 in the Journal of Clinical Oncology by Sharlene Gill et al. (7 studies: NCCTG, ECOG/NCCTG/INT, SWOG/INT0035, NCIC-CTG, FFCD, GIVIO) showed that the use of adjuvant chemotherapy after a radical resection increases the rate of a 5 -year DFS to 67\% (from 55\%) and 5-year OS to 71\% (from $64 \%$ ), in comparison with surgery alone; this translates into a relative reduction of recurrence risk (DFS) and the risk of death (OS) by $30 \%$ and $26 \%$ respectively [1]. These data however, concern, first of all stage III colon cancer patients (pN1-pN2b) as defined according to TNM/AJCC. The problem arises when an oncologist must make a decision about the treatment of a stage II patient (pT3-pT4b and pN0).

Two prospective trials (NACCP - Taal BG et al. 2001, Quasar - Gray R et al. 2007) showed an improvement in the 5-year survival, at a level of 3-8\%, resulting from the application of adjuvant chemotherapy (in comparison with a group of patients undergoing surgery alone) in a group of patients with stage II colon cancer [1].

Some other studies (e.g. IMPACT B2 - Fang SH et al. 2014, Cancer Care Ontario — Figueredo A et al. 2004, SEER/ MEDICARE - O'Connor ES et al. 2011) did not confirm the prolongation of overall survival in the adjuvant chemotherapy arm as compared with surgery alone, yet these studies showed an improvement of DFS by 3\% (Impact B2) and by 5-10\% (Cancer Care Ontario) in comparison with surgery alone [1].

It must be strongly emphasised that the collective negative analyses were drafted on the basis of the "old" cli- nical trials. Additionally, in the largest collective negative overview, Cancer Care Ontario from 2011 (37 trials and 11 meta-analyses), concerning a group of 20,317 patients with stage II according to AJCC, 5-FU/FA was used only in 2082 cases (which makes up about 10\%), whilst the remaining 18,235 patients (about $90 \%$ ) received nonoptimal adjuvant chemotherapy, e.g. 5-FU without biomodulation or oral 5-FU (only $10-40 \%$ of the administered dose is absorbed) or 5-FU+ semustine or 5-FU+ levamisole (increased myelotoxicity 5-FU and chemotherapy is discontinued more often) or unspecific immunotherapy or intraperitoneal 5 -FU infusions or 5-FU infusions into the hepatic artery, which means that an improvement in the survival of the patients in the arm with adjuvant chemotherapy could not take place. In a retrospective analysis, SEER/MEDICRE only 3834 (about 20\%) of stage II patients in the group of increased risk received adjuvant chemotherapy (out of 18,613 analysed patients) and, additionally, they were not randomised, as the decision to include chemotherapy depended on the oncologist; moreover, within the chemotherapy group, the left-sided colon location of cancer was dominating (currently it is known that in this location, in 40-50\% cases the CMS2 molecular subtype dominates - and it has better prognoses and longer OS and RFS) [1, 3].

In my opinion, the above negative meta-analyses and collective studies, with regards to some substantive drawbacks (e.g. suboptimal adjuvant chemotherapy in the adjuvant therapy arm, the selection of patients with better prognoses etc.) do not contradict the statement that stage II colon cancer patients do not benefit from adjuvant chemotherapy.

The opponents of adjuvant treatment in II stage of colon cancer also raise the issue of the toxicity of 5-FU- based chemotherapy, especially among persons above 70 years of age, referring to frequent events of stomatitis and leucopoenia as well as diarrhoea. It was proven that the G3 and G4 toxicity (according to CTCAE criteria) of 5-FU chemotherapy increases the risk of death of a patient by about $1 \%$ - so such a low level of toxicity can be accepted [1].

The recently published analysis from the National Cancer Data Base from 2016 (Casadaban L. et al. [4]), covering 153 110 II stage colon cancer patients (including 31,782 in the chemotherapy arm), showed that the benefits from adjuvant chemotherapy such as an improvement in the overall survival $(p<0.001)$ are gained by the patients in the group of high and low risk of recurrence, and this is irrespective of their age ( $<65$ years of age and $>65$ years of age). In 2015 (Böckelman C. et al. [5]) an analysis was published on the basis of data concerning 1697 patients treated in 1999-2008 in British Columbia (1286 $=76 \%$ patients in the high risk group and $411=24 \%$ low risk patients, out of whom $373=$ $29 \%$ and $51=12 \%$ respectively received adjuvant therapy), in which the benefit in OS was proven among the high risk 
colorectal cancer patients treated with adjuvant therapy prolongation of RFS, DSS and OS concerned the patients with pT4 stage. A similar result was rendered by an analysis published in 2016 on the basis of the data from the national cancer register in the Netherlands (Verhoeff SR et al. [6]). It was carried out on a population of 4940 II clinical stage colorectal cancer patients with clinical features of high risk (out of 10,935 colorectal cancer patients in clinical stage II in whose case the diagnosis was established in 2008-2012 and who were treated surgically) — the benefits in adjuvant therapy were observed solely in the patients with pT4 stage - the risk of death in patients in the pT4 stage receiving chemotherapy was lower in comparison with patients who did not receive chemotherapy: the 3-year survival rate was $91 \%$ and $73 \%$ respectively, HR $0.43,95 \% \mathrm{Cl} 0.28-0.66$.

The use of adjuvant chemotherapy in II stage colon cancer may be determined either by clinical and pathological factors or by clinical and molecular factors. The first group of factors comprises: 1) pT4 stage of cancer advancement (the most important factor); 2) inadequate scope of lymphadenectomy (the lack of lymph nodes assessment in the post-operative specimen or the number of resected and assessed lymph nodes lower than 12); 3) G3 cancer grade and small-cell cancer; 4 ) the involvement of the peritumoral lymph and blood vessels; 5) R1 resection; 6) patients operated on account of an intestinal perforation or ileus. The second group of factors comprises the evaluation of microsatellite instability (MSI), which is the marker of the genetic mechanism of mismatch repair (MMR - mutator genes - e.g. $M L H 1, M S H 2$ ) and the BRAF gene mutation. Depending on the rate of mismatch microsatellite regions, detected in specific tests, the cancer can be classified as characterised with MSI-high (MSI-H) or MSI-low (MSI-L) [1, 8].

Microsatellite instability is a feature characteristic of not only hereditary nonpolyposis colorectal cancer (Lynch syndrome, HNPCC), but it also occurs in morphological-molecular subtypes of sporadic cancer, in which carcinogenesis comprise an epigenetic disorder consisting of silencing one or more MMR genes - most frequently the MLH1 gene. MMR deficiency (dMMR), the main marker of which is MSI-H - is found in about $20 \%$ of patients with sporadic colorectal cancer. It must be pointed out that in colon cancer, dMMR is connected with better prognosis and a worse response to 5-FU-based chemotherapy (Tougeron D et al. [8]).

The type of cancer in which MSI is not found, is referred to as a microsatellite stable (MSS) or a MMR proficient (pMMR). In the morphological-molecular subtype (which is the most frequent in sporadic colorectal cancer), usually chromosomal instability (CIN) is found at the level of the loss of heterozygosity (LOH), most often in 18q, 2p, 5q, 17p. Chromosomal instability is an independent prognostic survival marker in stage II and III colorectal cancer (according to TNM/AJCC) with respect to DFS $(p<0.001)$ and OS $(p<0.0076)$ [9].
In a collective analysis of a few trials, such as, among others: Pan-European Trials in Adjuvant Colon Cancer (PETACC III), the European Organization for Research and Treatment of Cancer (EORTC) 40993 and the Swiss Group for Clinical Cancer Research (SAKK), a molecular marker, such as MSI-H, was found in $22 \%$ of stage II colon cancer patients. The multivariate analysis showed better prognosis in patients with stage II MSI-H cancer with regards to the improvement of the overall survival (OS) and RFS in comparison to patients with MSS type cancer ( $H R=0.45 ; p=0.0003)$. In the stage II cancer patients (according to pTNM/AJCC) in the MSI-H subgroup, the BRAF gene mutations were dominant $(63 \%)$, but only in the MSI-L and MSS subgroups did the presence of $B R A F$ mutations result in a shorter overall survival. This led the authors to the conclusion that MSI-H may render a "protective effect" for recurrence in relation to the negative effect of the BRAF mutation [10].

On the basis of the results of the above studies, one can assume that the presence of MSI-H in stage II colon cancer has a positive prognostic significance. Nevertheless, the data from the American register, SEER-NCl, show that 5-year survival in patients with stage IIC colon cancer is much worse (37\%) than in the case of stage IIIA (T1-2N1/T1N2a - 73\%), and close to stage IIIB (pT3-T4aN1/ pT2-T3N2a/pT1-T2N2b $-45 \%$ ) [2]. Therefore it is beyond all doubt that among the patients with stage II C colorectal cancer, there are cases of MSI-H, which should definitely be treated with adjuvant chemotherapy.

However, today the majority of oncologists, when qualifying patients to adjuvant therapy, base their decisions on clinical and pathological parameters; whilst these parameters "tend to" pick a small number of patients with stage II colon cancer who should be treated with adjuvant therapy, they are unable at the same time to determine the degree of recurrence risk in an individual case. O'Connell et al [11] in 2010 publish the best - in my opinion to date - multivariate analysis of the dependence of gene panel expression which seem to be connected with the risk of recurrence (RSG - recurrence score gene panel) and with response to 5-FU/FA adjuvant therapy (TSG —-treatment score gene panel). The RNA extracted from paraffin blocks in 1581 patients with colorectal cancer in stages II and III according to PTNM/AJCC, was studied with the reverse polymerase chain reaction test (RT-qPCR). Then the RSG panel consisting of 12 genes ( 7 cancer genes +5 reference genes) and TSG panel consisting of 11 genes ( 6 genes of the 5 -FU response +5 reference genes) were determined and the risk of colorectal cancer recurrence and benefit from the use of 5-FU/ FA adjuvant chemotherapy were calculated with statistical methods. On the basis of these calculations, the algorithms for evaluation of the risk of recurrence and benefits from chemotherapy were constructed and they were used for the identification of the groups of low, intermediate and high 
recurrence risk as well as the benefits in the administration of 5-FU/FA adjuvant chemotherapy. The cancer recurrence score (RS) was calculated with the cut-off thresholds for the risk groups in stage II being: low $\mathrm{RS}<30$, intermediate RS 31-40 and high RS $\geq 41$, which translated into a 3-year cancer recurrence risk of $8 \%, 11 \%$ and $25 \%$ patients. The difference between the low and intermediate groups was not statistically significant. The cut-off thresholds for the benefits in the use of 5-FU/FA adjuvant chemotherapy, after 3 years were, in the low, intermediate and high risk groups: (-)3\%, 3\% and $8 \%$ respectively $[11,12]$.

In Polish conditions, the evaluation of the recurrence score (RS) is rarely possible, and that is why the author of this paper proposed a simplified model for qualification of adjuvant therapy in stage II colon cancer patients (according to pTNM/AJCC). This model is based on the analysis of the MSI BRAF gene mutation as well as the T3-pT4 stage and lymphadenectomy scope. Figure 1 presents a modified treatment algorithm which takes into consideration these 4 parameters and should be of some assistance in taking decisions about the application of adjuvant chemotherapy.

In accordance with Figure 1 and assuming a minimum positive effect of chemotherapy on the level of 3-8\%, stage II colon cancer patients with a low recurrence risk (IIA/IIB MSI-H irrespectively of the BRAF gene mutation or IIA MSI-L/MSS and optimum lymphadenectomy and no BRAF mutation) do not require adjuvant chemotherapy at all. The stage II colon cancer patients with a high recurrence risk in turn (IIA/IIB MSI-L/ MSS and inadequate lymphadenectomy and BRAF mutation) will benefit from the adjuvant use of 5-FU/FA or capecitabine. In the case of post-operative diagnosis of colon cancer IIA/IIB MSI-L/MSS with adequate scope of lymphadenectomy and $B R A F$ mutation, and IIA MSI-L/MSS with inadequate lymphadenectomy scope and lack of BRAF mutation, the decision to apply adjuvant chemotherapy should be taken by a clinical oncologist, taking into consideration other factors (such as age, comorbidities, other existing clinical or pathological risk factors etc.) and - respecting the decision of the patient the author of this paper, on the basis of his own experience is convinced of the appropriateness of the use of adjuvant chemotherapy in this group of patients with an intermediate risk of recurrence. Stage IIC colon cancer patients should be given a 2-drug regimen - FOLFOX or XELOX $[1,13]$.

In 2015 the characteristic features for molecular subtypes of colorectal cancer were published (CMS - The consensus molecular subtypes of $C R C)[3,14]$. These criteria are summarised in Table I.

A routine analyses of the cases of colorectal cancer (colon cancer and rectal cancer) carried out according to the CMS molecular criteria will allow for a more precise determination of the population of stage II patients who would benefit from adjuvant chemotherapy.

\section{Conclusions}

Analysing the problem with a view to a risk of cancer recurrence as well as DFS and OS prolongation with the use of adjuvant chemotherapy, stage II colon cancer patients (according to pTNM/AJCC) make up a heterogenous group of patients.

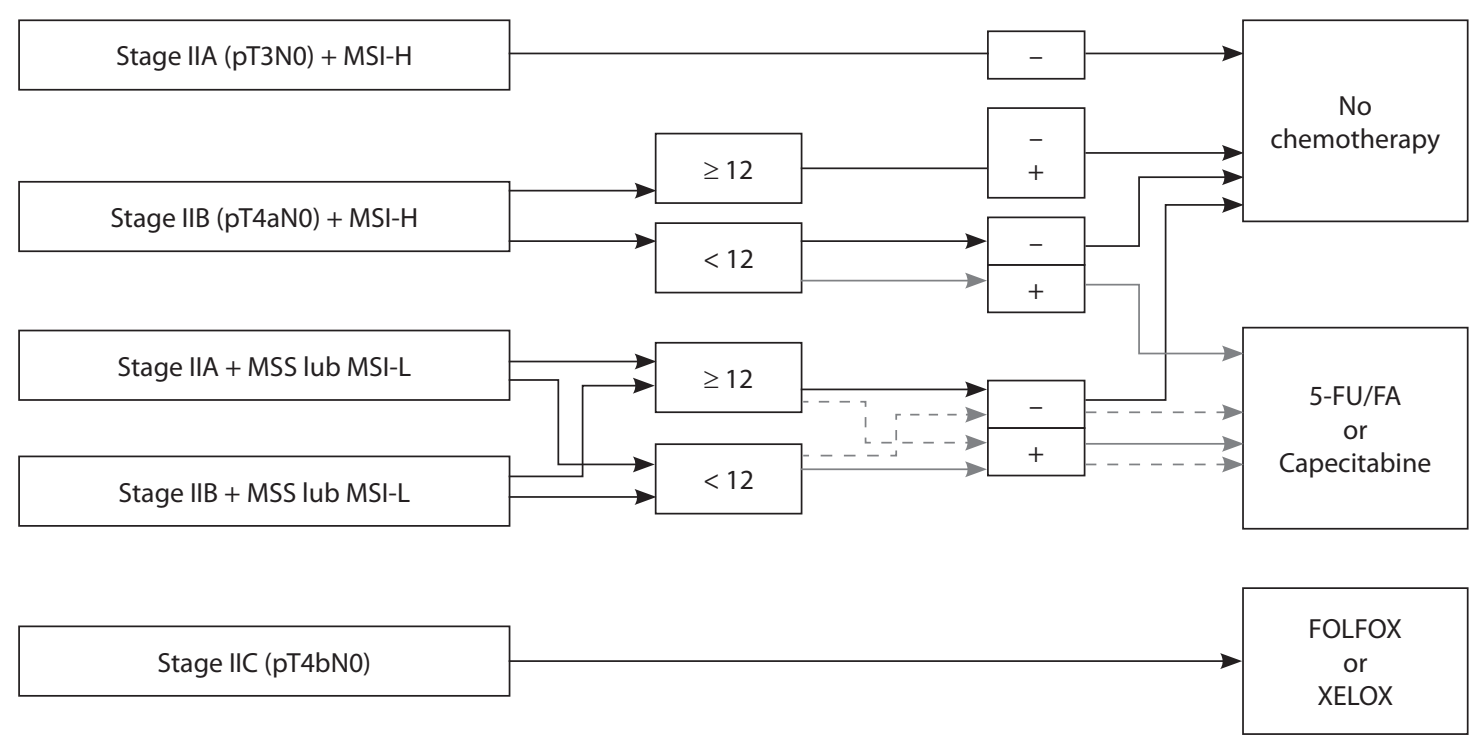

MSI - microsatelite instability (dMMR); MSS - microsatelite stable (pMMR). $\quad---\rightarrow$ up to the decision of the clinical oncologist

Figure 1. A modified algorithm helpful for the selection of adjuvant chemotherapy in patients with stage II colon cancer as defined by pTNM/AJCC 
Table I. Molecular and clinical features of colorectal cancer according to the CMS classification and the possibilities of studies of the personalised therapy suggested on the basis of this classification

\begin{tabular}{|c|c|c|c|c|}
\hline & $\begin{array}{l}\text { CMS1 (MSI Immune - } \\
\text { MSI immunogenic) }\end{array}$ & $\begin{array}{l}\text { CMS2 (Canonical - basic } \\
\text { epithelial) }\end{array}$ & $\begin{array}{l}\text { CMS3 (Metabolic } \\
\text { - connected with } \\
\text { the deregulation of } \\
\text { metabolic pathways) }\end{array}$ & $\begin{array}{l}\text { CMS4 (Mesenchymal } \\
\text { - micro-environment- } \\
\text { dependent) }\end{array}$ \\
\hline Prevalence & $\sim 15$ & $\sim 40$ & $\sim 15$ & $\sim 30$ \\
\hline $\begin{array}{l}\text { Tumour location within the } \\
\text { large intestine }\end{array}$ & $\begin{array}{l}\text { Right-side of colon } \\
\text { location is dominating }\end{array}$ & $\begin{array}{l}\text { Left-side of colon } \\
\text { and anal location is } \\
\text { dominating }\end{array}$ & $\begin{array}{l}\text { Right-side of colon } \\
\text { location is dominating }\end{array}$ & $\begin{array}{l}\text { Left-side of colon } \\
\text { and anal location is } \\
\text { dominating }\end{array}$ \\
\hline Cancer cells features & $\begin{array}{l}\text { Hyper-mutated and } \\
\text { hyper-methylated, MSI, } \\
\text { dominance of mutated } \\
\text { BRAF }\end{array}$ & $\begin{array}{l}\text { CIN, MSS, EGFR+, } \\
\text { excessive ERRB2 } \\
\text { regulation }\end{array}$ & $\begin{array}{l}\text { Mixed MSI/MSS, CIN, } \\
\text { deregulation of metabolic } \\
\text { pathways of mutated } \\
\text { KRAS }\end{array}$ & $\begin{array}{l}\text { MSS, CIN, disordered } \\
\text { interactions between } \\
\text { epithelial and stromal } \\
\text { cells }\end{array}$ \\
\hline $\begin{array}{l}\text { Microenvironment cell } \\
\text { properties }\end{array}$ & $\begin{array}{l}\text { Numerous cytotoxic and } \\
\text { helper lymphocytes T and } \\
\text { NK cells }\end{array}$ & $\begin{array}{l}\text { Small infiltrations of } \\
\text { immunological and } \\
\text { stromal cells }\end{array}$ & $\begin{array}{l}\text { Small infiltrations of } \\
\text { immunological and } \\
\text { stromal cells }\end{array}$ & $\begin{array}{l}\text { Numerous stromal cells, } \\
\text { numerous suppressor } \\
\text { cells T-reg. Iymphocytes, } \\
\text { B lymphocytes, myeloid- } \\
\text { derived cells }\end{array}$ \\
\hline Prognosis & $\begin{array}{l}\text { Longer RFS, after } \\
\text { recurrence shorter OS }\end{array}$ & Longer RFS and OS & Longer RFS and OS & Shorter RFS and OS \\
\hline $\begin{array}{l}\text { Medication to be used in } \\
\text { clinical trials }\end{array}$ & $\begin{array}{l}\text { PD-1 and PD-L1 } \\
\text { inhibitors; BRAF signalling } \\
\text { pathway inhibitors }\end{array}$ & $\begin{array}{l}\text { Signalling pathway } \\
\text { inhibitors through EGFR, } \\
\text { inhibiting overexpression } \\
\text { of proteins from the HER } \\
\text { family }\end{array}$ & $\begin{array}{l}\text { PanRAF and MEK } \\
\text { signalling pathway } \\
\text { inhibitors in association } \\
\text { with inhibitors of } \\
\text { enzymatic paths }\end{array}$ & $\begin{array}{l}\text { Associating immune- } \\
\text { stimulating medication } \\
\text { with immunosuppression }\end{array}$ \\
\hline
\end{tabular}

I believe that adjuvant chemotherapy should be provided for every patient in the very high risk (IIC) and high risk group (IIA/IIB MSI-L/MSS and inadequate lymphadenectomy and $B R A F$ mutation), but also from the group of intermediate risk of cancer recurrence (IIA/IIB MSI-L/MSS with adequate scope of lymphadenectomy and BRAF mutation and IIA MSI-L/MSS with inadequate lymphadenectomy and lack of BRAF mutation) - upon obtaining the patient's informed consent. If the oncologist does not have any possibility to obtain the results of the microsatellite instability test or BRAF_gene mutation, it would be advisable to transfer the patient to a centre which has access to such a test through which adjuvant chemotherapy could optimally be used. This will definitely translate into an improvement of the treatment results for colon cancer patients.

Conflict of interest: none declared

\section{Prof. Andrzej Deptała, MD, PhD}

Central Clinical Hospital of the MSWiA, Warszawa, Poland,

Clinical Department of Oncology and Hematology,

Medical University of Warsaw

Department of Cancer Prevention

ul. Żwirki i Wigury 6

02-091 Warszawa, Poland

e-mail:andrzej.deptala@wum.edu.pl

\section{Received \& Accepted: 5 Feb 2018}

Based on the presentation at the V Annual Conference of the Nowotwory Journal of Oncology, 'Oncological Debates', held in Warszawa, 7-8th April 2017

\section{References}

1. Deptała A, Dudzisz-Śledź M. Leczenie adiuwantowe raka jelita grubego. In: Rak jelita grubego. Deptała A, Wojtukiewicz MZ (red.). Poznań: Termedia Wydawnictwa Medyczne, 2017: 253-273.

2. AJCC cancer staging manual. Amin MB, Edge $S$, Greene $F$ et al. (eds.). Chicago: American Joint Comitte on Cancer; Springer Int Publ AG, Switzerland, 2017: 251-284.

3. McCleary NJ, Benson AB 3rd, Dienstmann R. Personalizing adjuvant therapy for stage II/III colorectal cancer. ASCO Educational Book, 2017; 37: 232-245.

4. Casadaban L, Rauscher G, Aklilu M et al. Adjuvant chemotherapy is associated with improved survival in patients with stage II colon cancer. Cancer 2016; 122: 3277-3287.

5. Böckelman C, Engelmann BE, Kaprio T et al. Risk of recurrence in patients with colon cancer stage II and III: a systematic review and meta-analysis of recent literature. Acta Oncol 2015; 54: 5-16.

6. Verhoeff SR, van Erning FN, Lemmens VE et al. Adjuvant chemotherapy is not associated with improved survival for all high-risk factors in stage II colon cancer. Int J Cancer 2016; 139: 187-193.

7. Kumar A, Kennecke HF, Renouf DJ et al. Adjuvant chemotherapy use and outcomes of patients with high-risk versus low-risk stage II colon cancer. Cancer 2015; 121: 527-534.

8. Tougeron D, Mouillet G, Trouilloud I et al. Efficacy of adjuvant chemotherapy in colon cancer with microsatellite instability: a large multicenter AGEO Study. J Nat/ Cancer Inst 2016; 108. doi: 10.1093/jnci/djv438.

9. Watanabe T, Kobunai T, Yamamoto $\mathrm{Y}$ et al. Chromosomal instability (CIN) phenotype, CIN high or CIN low, predicts survival for colorectal cancer. J Clin Oncol 2012; 30: 2256-2264.

10. Roth AD, Tejpar S, Delorenzi $M$ et al. Prognostic role of KRAS and BRAF in stage II and III resected colon cancer: results of the translational study on the PETACC-3, EORTC 40993, SAKK 60-00 Trial. J Clin Oncol 2010; 28: 466-474.

11. O'Connell MJ, Lavery I, Yothers $\mathrm{G}$ et al. Relationship between tumor gene expression and recurrence in four independent studies of patients with stage II/III colon cancer treated with surgery alone or surgery plus adjuvant fluorouracil plus leucovorin. J Clin Oncol 2010; 28: 3937-3944.

12. Niedzwiecki D, Frankel WL, Venook AP et al. Association between results of a gene expression signature assay and recurrence-free interval in patients with stage II colon cancer in Cancer and Leukemia Group B 9581 (Alliance). J Clin Oncol 2016; 34: 3047-3053.

13. Påhlman LA, HohenbergerWM et al. Should the benefit of adjuvant chemotherapy in colon cancer be re-evaluated? J Clin Oncol 2016;34:1297-1299.

14. Muller MF, Ibrahim AE, Arends MJ. Molecular pathological classification of colorectal cancer. Virchov Arch 2016; 469: 125-134. 